\title{
Co-occurring Conditions and Health-Related Quality of Life in Patients With Bipolar Disorder
}

Amy M. Kilbourne, PhD, Brian E. Perron, PhD, Briana Mezuk, PhD, Deborah Welsh, MS, Mark Ilgen, PhD, AND MARK S. BAUER, MD

\begin{abstract}
Objective: To assess changes in health-related quality of life (HRQOL) and to determine whether co-occurring substance use and medical comorbidities were associated with worse HRQOL over a 1-year period in a naturalistic sample of patients with bipolar disorder. Method: Patients enrolled in the Continuous Improvement for Veterans in Care: Mood Disorders (CIVIC-MD), a large prospective study from July 2004 to July 2006, completed baseline and follow-up assessments related to bipolar symptoms, comorbidity, and Short Form 12 HRQOL assessment at baseline and at 1 year later. Predictors of changes in HRQOL mental and physical health component scores were determined, using multivariable linear regression models and path analyses. Results: Of 334 participants, the mean age was 49 years (standard deviation $[\mathrm{SD}]=10.1$ years; range $=21-78$ years), $15.9 \%$ women, and $10.8 \%$ African American. At baseline, 19.8\% reported hazardous drinking, 25.5\% reported illicit drug use, and the mean number of co-occurring medical conditions was $2.70(\mathrm{SD}=2.02)$. Illicit drug use was associated with worse mental HRQOL $(\beta=-2.01$; $p<.05)$, and increased number of medical comorbidities was significantly associated with worse physical HRQOL over time $(\beta=$ $-0.71 ; p<.01)$. Depressive symptoms exhibited a strong and consistent influence on mental health scores over time, but path analyses revealed that they did not seem to mediate effects of comorbidities on lower HRQOL. Conclusions: Illicit drug use and medical comorbidity negatively affected mental HRQOL over time, independent of bipolar symptoms, suggesting the need for interventions that address physical health and drug treatment needs for patients with bipolar disorder. Key words: bipolar disorder, comorbidity, health-related quality of life.
\end{abstract}

SF-12 = Short Form 12 health-related quality of life assessment; HRQOL = health-related quality of life; PCS = physical health component score; MCS = mental health component score; CIVICMD = Continuous Improvement for Veterans in Care: Mood Disorders study; ISS = Internal State Scale; PTSD = posttraumatic stress disorder; RMSEA = root mean square error of approximation; TLI = Tucker-Lewis Index; CFI = comparative fit index.

\section{INTRODUCTION}

B ipolar disorder is one of the top ten most disabling conditions and is associated with substantial functional impairment $(1,2)$ and healthcare costs $(3,4)$. This illness is characterized by alternating manic and depressive episodes and is estimated to affect up to $5.5 \%$ of the U.S. population (5). When untreated, bipolar disorder can increase the risk of co-occurring medical and substance use disorders $(6,7)$, frequent hospitalizations (8), and adverse outcomes including premature mortality (9).

Not surprisingly, health-related quality of life (HRQOL) in patients with bipolar disorder (8) has been reported to be lower than in the general population, even among those not reporting current manic or depressive episodes $(8,10)$, and

From the VA Ann Arbor Health Services Research \& Development and National Serious Mental Illness Treatment Research and Evaluation Center (A.M.K., B.E.P., B.M., D.W., M.I.), Ann Arbor, Michigan; Department of Psychiatry (A.M.K., M.I.), University of Michigan, Ann Arbor, Michigan; University of Michigan School of Social Work (B.E.P.), Ann Arbor, Michigan; Department of Epidemiology (B.M.), University of Michigan School of Public Health, Ann Arbor, Michigan; Harvard South Shore Psychiatry Residency Training Program (M.S.B.), Harvard Medical School, Boston, Massachusetts; and VA Boston Healthcare System (M.S.B.), Boston, Massachusetts.

Address correspondence and reprint requests to Amy M. Kilbourne, VA Ann Arbor HSRD/SMITREC (11H), 2215 Fuller Road, Ann Arbor, MI 48105. E-mail: amykilbo@umich.edu

Received for publication January 27, 2009; revision received May 13, 2009.

This research was supported by Grants IIR 02-283 (A.M.K.) and IIR 07-115 (A.M.K.) from the Department of Veterans Affairs, Veterans Health Administration, Health Services Research and Development Service. The views expressed in this article are those of the authors and do not necessarily represent the views of the Department of Veterans Affairs.

DOI: 10.1097/PSY.0b013e3181b49948 among those with bipolar disorder Type II (11). HRQOL is an important indicator of current functioning and prognosis $(12,13)$, and low HRQOL is consistently associated with increased risk of mortality across several chronic conditions $(14,15)$ including mental disorders $(16)$.

Achieving symptom remission is considered one of the essential treatment goals for persons with bipolar disorder. However, there is a growing realization that these individuals experience a substantial prevalence of co-occurring substance use and medical disorders $(7,17)$, which can also adversely affect symptom remission and contribute to poor outcomes including lower HRQOL. The few observational studies characterizing physical and mental HRQOL in patients with bipolar disorder have been cross-sectional in nature $(2,8,18,19)$, limited to selective randomized, controlled trials (20), or have not controlled for co-occurring medical or substance use disorders (21). Understanding the extent to which co-occurring conditions contribute to worsening symptomatology and subsequent HRQOL over time within a naturalistic treatment setting is central to the process of tailoring treatment strategies for patients with this illness (22).

The goal of this study is to assess changes in HRQOL over a 1-year period and to determine whether co-occurring conditions were associated with lower HRQOL independent of bipolar symptomatology, based on a naturalistic cohort of patients with bipolar disorder. We hypothesized that co-occurring substance use and medical conditions will be associated with decreased physical and mental HRQOL scores in 12 months, after controlling for other patient factors, and this effect will be mediated by increased manic and depressive symptoms.

\section{METHODS}

This is a prospective, longitudinal cohort study based on the Continuous Improvement for Veterans in Care: Mood Disorders (CIVIC-MD) study, designed to identify the predictors of poor outcomes among individuals with bipolar disorder. Patients from a large Veterans Affairs (VA) medical center in the mid-Atlantic region, who were diagnosed and treated for bipolar disorder based on medical record review and confirmation of diagnosis from 


\section{QUALITY OF LIFE IN BIPOLAR DISORDER}

the patient's mental health clinician between July 2004 and July 2006, were consented, enrolled, and asked to complete a baseline and 1-year follow-up survey that included measures of demographic characteristics, substance use history, manic and depressive symptoms, treatment preferences, and HRQOL. Additional data on co-occurring medical conditions were ascertained from VA claims data. Patient inclusion criteria included current recorded diagnosis of bipolar disorder (Type I or II) confirmed by their provider. Exclusion criteria included unstable acute medical conditions, acute psychiatric symptoms (defined as current manic or psychotic episode at the time of enrollment that precluded informed consent as determined by their mental healthcare provider), or significant cognitive impairment that precluded informed consent. Further details regarding CIVIC-MD are available elsewhere (23). This study was reviewed and approved by local Institutional Review Boards, and all subjects provided their informed consent.

\section{Measures}

Our primary outcome, HRQOL, was assessed using the Short Form (SF-12) measure in the baseline and 1-year follow-up surveys (24). As with the longer version of this HRQOL assessment (SF-36), the SF-12 is a self-reported, 12-item assessment that ascertains the impact of physical and mental symptoms on the ability to function in a series of daily tasks, vigor, pain, and one's self-perceptions of overall health compared with others. The SF-12 generates two scores - the mental health component score (MCS) and physical health component score (PCS). The MCS and PCS are highly correlated with the longer SF-36 summary scores for MCS and PCS ( $r=0.92$ and 0.91 , respectively) (24).

Bipolar disorder symptoms were ascertained at baseline and follow-up, using the Internal State Scale (ISS), a 15-item self-completed instrument that assesses bipolar disorder symptom severity (25). Two symptom subscales were created based on the ISS, representing symptoms of mania and depression, respectively. Each subscale is comprised of a set of symptoms, and the patients self-reported the degree to which they experienced each symptom, using a Likert scale (from $0=$ "not all all/rarely" to $100=$ "very much so"). For the mania subscale, possible scores range from 0 to 50 points, with a higher score indicating more severe manic symptoms. For the depression subscale, scores range from 0 to 20 points, with higher scores indicating more severe depressive symptoms. The ISS has a test-retest reliability exceeding 0.8 and prior research indicated that these subscales are highly correlated with clinician ratings of mania and depression (25).

Co-occurring conditions were estimated, using measures for substance use disorders, including alcohol (hazardous or binge drinking) and illicit drug use ascertained from the patient baseline survey, and general medical conditions ascertained from medical record data. Hazardous (binge) drinking was defined as whether the patient reported having $>5$ drinks on one occasion at any time within the past year (26). This assessment was strongly correlated with clinician assessments of alcohol use disorders (sensitivity $=0.95$; specificity $=0.69$ ). Any current illicit drug use (defined as marijuana, cocaine, methamphetamines or other stimulants, heroin or other opioids, hallucinogens, ecstasy, and sedatives/hypnotics) was ascertained based on questions regarding the past year, using Composite International Diagnostic Interview Short Form (27). Compared with full-length Diagnostic Interview Survey assessments for drug use disorders, the sensitivity of the Composite International Diagnostic Interview Short Form was $92 \%$ and the specificity was $91 \%$.

Co-occurring medical conditions were ascertained from the VA electronic medical record data. Medical diagnosis data were ascertained on or within 1 year before the enrollment date based on International Classification of Diseases-9th Revision (ICD-9 CM) diagnostic codes, categorized by organ system using a previously established method (19). We also assessed for the presence of current anxiety symptoms (yes/no) based on a screener question from the Patient Health Questionnaire (28) and posttraumatic stress disorder (PTSD), based on VA electronic medical record data.

Other patient sociodemographic information (age, race, sex, marital status, living alone or not, and college education or less than a college education) were assessed, using the baseline survey questions. Bipolar subtype (Type I or Type II) from provider confirmation was also ascertained.

\section{Analyses}

Two analytic approaches were used to test our hypothesis that the association between co-occurring conditions and decreased physical and mental HRQOL is explained (mediated) by increased manic and depressive symptoms over the 12 -month period. First, we employed multivariate linear regression to assess the longitudinal predictors of changes in HRQOL physical and mental health scores over the 1-year period. Second, we used path analysis to detail to what extent bipolar symptoms explained decreases in physical and mental health HRQOL independent of co-occurring conditions.

\section{Multivariate Linear Regression}

Change scores were created for the SF-12 physical (PCS) and mental health (MCS) composite scores as well as for the depressive and manic symptom scales based on the baseline and 1-year follow-up surveys. Two linear regression models for each outcome (MCS and PCS) were run to determine the independent effect of co-occurring substance use and medical disorders on SF-12 change scores, and whether manic and depressive symptom change scores explained these changes. The first model controlled for patient demographics (age, race, gender, live alone, married, college educated), SF-12 baseline score, and co-occurring conditions at baseline (binge drinking, illicit drug use, number of medical comorbidities diagnosis of PTSD, diagnosis of bipolar disorder Type II (versus Type I), and presence of anxiety symptoms and PTSD diagnosis). The second (full) model added the effect of manic and depressive symptom scores at baseline and symptom change scores. Adjusted $\mathrm{R}^{2}$ values were used to assess regression model fit.

\section{Path Analysis}

Path models were used confirm our hypotheses by testing the direct and indirect pathways linking comorbidities and manic/depressive symptoms to HRQOL. An advantage of path analysis is the capability examining depression and mania within a set of temporally ordered and feedback relationships, which is not possible with multivariate linear regression. To achieve a parsimonious model, the primary independent variables (depression and mania), primary outcome variables (i.e., mental and physical HRQOL), and a subset of control variables that were statistically significant in the regression analyses were included. Path models were tested, using Mplus software (version 4.2) with maximum likelihood estimation. Model fit was based on the $\chi^{2}$ test and goodness-of-fit statistics, the root mean square error of approximation (RMSEA), Tucker-Lewis Index (TLI), and comparative fit index (CFI).

\section{RESULTS}

Overall, 720 patients were screened at the facility; of those, 616 patients were deemed eligible for participation. Of the 616 patients, 468 (76\%) were enrolled between July 2004 and July 2006. The remaining patients were not enrolled due to a lack of time for them to complete the survey (e.g., due to a pending outpatient appointment). Of the 468 patients, 435 (92\%) completed the baseline survey and 334 (77\%) completed the follow-up survey. Those who completed both the baseline and follow-up surveys were similar in demographic and clinical characteristics to those who did not complete the follow-up survey, with a few exceptions. The mean age for both groups was 49 years and $16 \%$ of those completing both the baseline and follow-up surveys were women versus $10 \%$ not completing the follow-up survey. In addition, comparing completers and noncompleters, the mean number of comorbidities was 2.6 and 2.9 , respectively.

Baseline manic and depressive symptom scores were also similar between the two groups: for mania, the scores were 19.9 and 18.2, respectively; for depression, scores were 7.9 and 7.2, respectively. HRQOL scores at baseline were also 
similar, with PCS scores 37.9 and 37.6, respectively, and MCS scores 31.7 and 32.0, respectively. The prevalence of anxiety symptoms was also similar between the two groups: $68 \%$ versus $72 \%$, respectively. Significant differences in patient characteristics were only found for race/ethnicity: whites were less likely to complete the follow-up survey ( $69 \%$ versus $79 \%$ in the completer and noncompleter groups, respectively; $p<.05$ ). Moreover, those completing the survey were less likely to have an illicit drug use diagnosis $(25 \%$ versus $37 \% ; p<.05)$.

Table 1 reports demographic results for those completing both baseline and follow-up surveys $(n=334)$. The mean age of the sample was 49 years $(\mathrm{SD}=10.6$ years; range $=21-78$ years), $62(14 \%)$ was female, and 61 (14\%) were African American. Twenty-six percent had a diagnosis of bipolar disorder Type II, and the remaining sample had Type I. Over a third reported living alone, and $17 \%$ graduated from college. Twenty-one percent reported hazardous drinking, 28\% reported illicit drug use, and over two thirds reported anxiety symptoms at baseline (Table 1). The mean number of cooccurring medical conditions was $2.77(\mathrm{SD}=2.05)$ and mean manic and depressive symptoms scores were 18.6 $(\mathrm{SD}=$ 13.0) and 7.8 ( $\mathrm{SD}=6.2)$, respectively, at baseline. Baseline mean SF-12 PCS and MCS scores were $38.0(\mathrm{SD}=7.6)$ and

TABLE 1. Baseline Characteristics of Patients With Bipolar Disorder $(n=334)$

\begin{tabular}{|c|c|}
\hline \multicolumn{2}{|l|}{ Patient Characteristics } \\
\hline Age & $49.4 \pm 10.1$ \\
\hline Women & $53(15.9)$ \\
\hline \multicolumn{2}{|l|}{ Ethnicity } \\
\hline White & $265(79.3)$ \\
\hline African American & $36(10.8)$ \\
\hline Other & $33(9.9)$ \\
\hline Living alone & $122(36.5)$ \\
\hline Married & $108(32.3)$ \\
\hline \multicolumn{2}{|l|}{ Education } \\
\hline College graduate & $61(18.4)$ \\
\hline Some college & $158(47.6)$ \\
\hline Less than college & $113(34.0)$ \\
\hline Bipolar disorder type II & $85(25.9)$ \\
\hline Binge drinking & $66(19.8)$ \\
\hline Any illicit drug use & $85(25.5)$ \\
\hline Current anxiety symptoms & $228(68.3)$ \\
\hline PTSD diagnosis & $22(6.9)$ \\
\hline \# Medical comorbidities & $2.70 \pm 2.02$ \\
\hline \multicolumn{2}{|l|}{ Bipolar symptoms (Internal State Scale) } \\
\hline $\begin{array}{l}\text { Baseline manic symptom score } \\
\quad(\text { range }=0-50)\end{array}$ & $18.2 \pm 13.0$ \\
\hline $\begin{array}{l}\text { Baseline depressive symptom score } \\
\quad \text { (range }=0-20)\end{array}$ & $7.9 \pm 6.2$ \\
\hline \multicolumn{2}{|l|}{ Health-related quality of life (SF-12) } \\
\hline $\begin{array}{l}\text { Physical health composite score } \\
\quad(\text { range }=20-56)\end{array}$ & $38 \pm 7.6$ \\
\hline $\begin{array}{l}\text { Mental health composite score } \\
\quad(\text { range }=6-57)\end{array}$ & $32 \pm 7.6$ \\
\hline
\end{tabular}

Data are represented as means $\pm \mathrm{SD}$ or as $\mathrm{n}(\%)$.

$\mathrm{SD}=$ standard deviation; PTSD $=$ posttraumatic stress disorder; SF-12 = Short Form 12 health-related quality of life assessment.
$32.0(\mathrm{SD}=7.7)$, respectively, and these values were similar in the follow-up survey results (data not shown).

\section{Multivariate Linear Regression}

For SF-12 mental HRQOL, illicit drug use was associated with decreased mental HRQOL $(\beta=1.41 ; p<.05)$. There was no significant effect of medical comorbidities on mental HRQOL in the first multivariable regression model (Table 2). In the full regression model, increased depressive symptom scores were strongly associated with decreased MCS scores 1 year later $(\beta=-0.61 ; p<.001)$. Still, the effect of illicit drug use was significant after adjusting for manic and depressive symptoms $(\beta=-2.01 ; p<.05)$. Adding manic and depressive symptoms substantially improved model fit (adjusted $\mathrm{R}^{2}$ changes from .30 to .46 ), indicating that depressive symptoms explained much of the variance in mental health change scores. Other clinical factors including bipolar II diagnosis or PTSD diagnosis were not associated with changes in mental HRQOL.

TABLE 2. Bipolar Disorder Symptoms, Co-occurring Conditions, and Changes in Health-Related Quality of Life: Multivariable Linear Regression Results

\begin{tabular}{|c|c|c|c|c|}
\hline & \multicolumn{2}{|c|}{$\begin{array}{c}\text { Change in HRQOL } \\
\text { MCS Score }\end{array}$} & \multicolumn{2}{|c|}{$\begin{array}{c}\text { Change in HRQOL } \\
\text { PCS Score }\end{array}$} \\
\hline & Model I & Model II & Model I & Model II \\
\hline Adjusted $\mathrm{R}^{2}$ & .30 & .46 & .32 & .33 \\
\hline Intercept & $21.60^{* * *}$ & $30.49 * * *$ & $27.12^{\star \star *}$ & $28.51^{* * *}$ \\
\hline $\begin{array}{l}\text { HRQOL baseline } \\
\text { score }^{a}\end{array}$ & $-0.65^{\star * *}$ & $-0.80^{* * *}$ & $-0.56^{\star \star *}$ & $-0.57^{* * *}$ \\
\hline Age (continuous) & 0.01 & 0.01 & $-0.09^{*}$ & $-0.09^{*}$ \\
\hline Female & -0.80 & -1.13 & $-2.31^{*}$ & $-2.23^{*}$ \\
\hline African American & -1.64 & -2.01 & 1.07 & 1.12 \\
\hline Other minority & 1.82 & 0.27 & 2.21 & 1.98 \\
\hline Live alone & -0.48 & -0.36 & 0.08 & 0.13 \\
\hline Married & 0.22 & 0.14 & 0.64 & 0.66 \\
\hline $\begin{array}{l}\text { College education } \\
\text { or greater }\end{array}$ & 0.94 & 0.62 & -1.00 & -0.95 \\
\hline Bipolar II & 0.78 & 1.01 & 1.14 & 1.14 \\
\hline Binge drinking & -1.01 & -1.24 & -0.94 & -0.75 \\
\hline $\begin{array}{l}\text { Any illicit drug use } \\
\text { disorder }\end{array}$ & -1.41 & $-2.01^{\star}$ & $-1.75^{*}$ & $-1.72^{*}$ \\
\hline $\begin{array}{l}\text { \# Co-occurring } \\
\text { medical cond. }\end{array}$ & -0.06 & 0.07 & $-0.74^{\star *}$ & $-0.71^{* *}$ \\
\hline Anxiety symptoms & -0.49 & 0.29 & $-0.60^{* *}$ & -0.26 \\
\hline PTSD diagnosis & 0.21 & 0.40 & 0.93 & 1.03 \\
\hline $\begin{array}{l}\text { Depressive } \\
\text { Sx-baseline }\end{array}$ & & $-0.61^{\star * *}$ & & -0.11 \\
\hline Manic Sx-baseline & & 0.03 & & -0.01 \\
\hline $\begin{array}{l}\text { Depressive } \\
\text { Sx-change }\end{array}$ & & $-0.57^{\star \star \star}$ & & -0.12 \\
\hline Manic Sx-change & & -0.04 & & 0.01 \\
\hline
\end{tabular}

$* p<.05 ; * * p<.01 ; * * * p<.001$.

${ }^{a}$ For the MCS outcome model, MCS baseline score was added; for the PCS outcome model, baseline PCS score was added.

HRQOL $=$ healthy-related quality of life; MCS = mental health component score; PCS = physical health component score; Model I = patient demographics and comorbidities; Model II = added manic and depressive symptoms; cond.$=$ condition; $\mathrm{PTSD}=$ posttraumatic stress syndrome; $\mathrm{Sx}=$ symptoms. 


\section{QUALITY OF LIFE IN BIPOLAR DISORDER}

For the SF-12 PCS, increased number of co-occurring medical conditions was associated with decreased PCS over time $(\beta=-0.74 ; p<.01)$. The presence of anxiety symptoms was also associated with decreased PCS ( $\beta=-0.60$; $p<.01)$. After controlling for manic and depressive symptoms, PCS change scores were still significantly associated with number of co-occurring medical conditions, but not for anxiety symptoms (Table 2). Depressive symptoms may have explained the association between anxiety and PCS decline (Table 2). Older patients and women were also more likely to experience decreased SF-12 PCS scores over time, and these effects remained after controlling for manic and depressive symptoms. Having a bipolar II diagnosis or PTSD diagnosis was not associated with changes in physical HRQOL. Both the first and full models demonstrated good overall fit (adjusted $\mathrm{R}^{2}=.32$ and .33 , respectively). A relatively high $\mathrm{R}^{2}(>.30)$ for both models indicates that medical comorbidities explained much of the effect on HRQOL changes over time (Table 2).

\section{Path Analysis Results}

We first describe the path analysis setup and then the final path analysis results. Two path models were fit to the dataone model using MCS as the outcome and the other using PCS. For the MCS model, the primary independent variables (i.e., mania and depression) and other significant control variables based on the foregoing regression analyses (i.e., gender, age, co-occurring substance use, and medical conditions) were included. Binge drinking, although not statistically significant in the regression models, was also included in the model due to its high prevalence in the study sample and prior research showing its effect on mental health and physical functioning. Baseline variables had paths pointing directly to the MCS baseline scores. The control variables also pointed to the follow-up measures of mania and depression. However, these paths were nonsignificant and subsequently trimmed to reduce the complexity of the models and likelihood of overfitting the data. Paths from the baseline measure of PCS and follow-up measures of mania and depression pointed to the follow-up measure of PCS. Finally, a reciprocal relationship between mania and depression at follow-up was specified. The final PCS model was constructed in the same manner, with the PCS measures replacing all MCS measures.

The final path analysis models are depicted graphically in Figure 1. The MCS model exhibited a good fit with the data, based on a nonsignificant $\chi^{2}$ value $\left(\chi^{2}[20]=22.55, p=.31\right)$, an RMSEA value that was $<0.05$ (RMSEA $=0.02,95 \%$ Confidence Interval $[\mathrm{CI}]=0.00-0.06)$ and TLI and CFI values that were $>0.90(\mathrm{TLI}=0.99$; CFI $=0.99)$. As depicted in Figure 1A, baseline depression but not mania was associated with lower. Lower baseline MCS were also associated with being female, binge drinking, and drug use. Similarly, depression but not mania at 1-year follow up was associated with lower follow-up MCS. In the specified reciprocal relationship between depression and mania, depression exhibited a significant effect on mania, but mania did not have a significant effect on depression.

The physical health path model also exhibited a good fit with the data. The $\chi^{2}$ was statistically significant $\left(\chi^{2}[19]=\right.$ $27.51, p=.28)$. The RMSEA value was $0.02(95 \% \mathrm{CI}=$ $0.00-0.06$ ), and the TLI and CFI were both 0.99 . Lower baseline PCS were significantly associated with higher base-
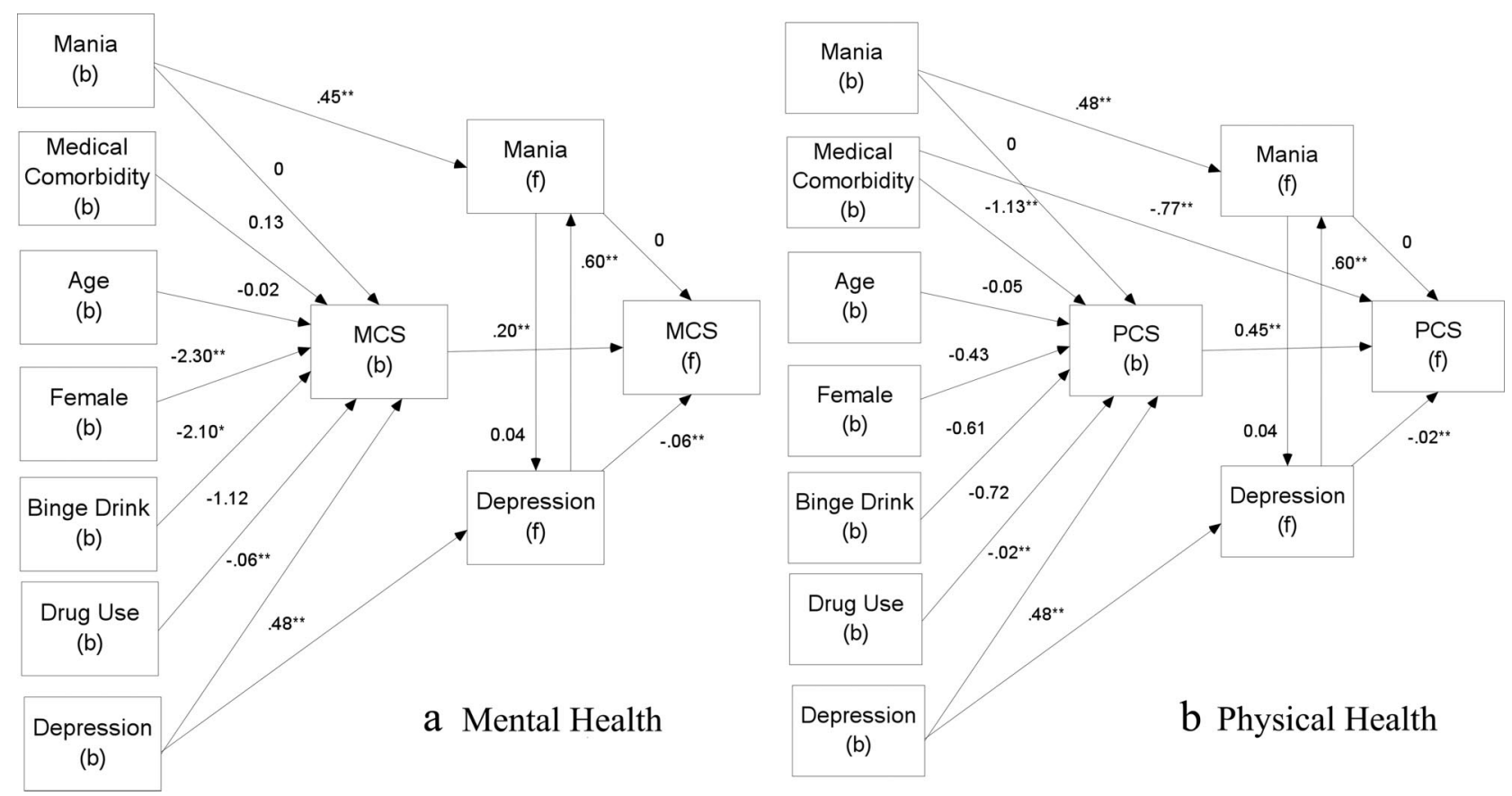

Figure 1. Path model representing health-related quality of life using baseline and 1-year follow-up data. a). Mental health-related quality of life; b). Physical health-related quality of life. ${ }^{*} p<.05 ;{ }^{* *} p<.01$; Medical Comorbidity $=$ number of co-occurring medical conditions; Mania $=$ manic symptom score; Depression $=$ depression symptom score. 
line depression scores and co-occurring medical conditions but none of the other baseline measures. Follow-up PCS were associated with follow-up depression and baseline co-occurring medical conditions, but not mania at follow-up or any of the other baseline measures (Fig. 1B). Again, similar to the mental health model, depression exhibited a significant influence on mania, but mania did not have a significant effect on depression.

\section{DISCUSSION}

The HRQOL in patients with bipolar disorder in this sample was significantly diminished, with scores that were on average 12 to 18 points lower than the national norm of 50 . Moreover, longitudinal regression and path analyses indicate that illicit drug use was associated with worse mental HRQOL, and co-occurring medical conditions were associated with worse physical HRQOL, independent of manic/ depressive symptoms or other patient factors.

The association between co-occurring medical conditions and physical HRQOL has been observed in prior cross-sectional studies of patients with bipolar disorder (19). Our path analyses confirmed an independent effect of baseline medical conditions on both baseline and follow-up physical HRQOL scores, independent of depressive symptoms, suggesting that medical comorbidity has a substantial impact on the functioning and well-being of patients with bipolar disorder. Similarly, the impact of illicit drug use on declining mental HRQOL scores remained after controlling for bipolar symptoms. Still, we were surprised to find that medical burden was not directly associated with decreased mental HRQOL. This may reflect increased coping or resilience among patients with chronic medical conditions.

Although depression exhibited a strong and consistent influence on both mental and physical health scores over time, it did not seem to mediate or explain away the effects of illicit drug use on decreased mental HRQOL, or medical comorbidities on decreased physical HRQOL over time. Still, the observed effects of co-occurring medical and substance use disorders on HRQOL were very consistent across regression and path analyses and highlight the importance of examining the relationship between symptoms and HRQOL over time.

We also observed no significant effect of PTSD diagnosis on changes in mental or physical HRQOL over time. Moreover, no significant difference was observed in HRQOL scores by bipolar subtype (I, II). Previous studies, notably Maina et al. found lower HRQOL scores in bipolar II compared with bipolar I patients $(11,17)$. In our study, bipolar subtype was not confirmed via structured clinical assessment, and hence, clinicians may have been unable to confirm bipolar subtype distinguished subtype in routine clinical care. We also found little effect of anxiety symptoms or PTSD diagnosis on HRQOL after controlling for other comorbidities. Albert et al. (29) found that, although the current prevalence of anxiety disorders was similar between patients with bipolar I and II, the negative association between anxiety disorder and HRQOL subscale scores was primarily observed in those with bipolar I disorder. Because we only screened for symptoms, further longitudinal research on the impact of anxiety disorder on functioning and outcome among patients with bipolar disorder is warranted.

To our knowledge, this is the first longitudinal study of HRQOL in bipolar disorder. Prior studies of HRQOL in bipolar disorder have been cross- sectional in nature and did not show whether mood states or symptoms lead to changes in HRQOL over time $(17,29)$. Advantages to our current study included the availability to examine longitudinally the mediating factors of poor HRQOL using path analysis, and consideration of co-occurring medical and substance use disorders.

These results highlight the importance of considering the impact of co-occurring conditions on HRQOL among patients with bipolar disorder. Co-occurring substance use and general medical conditions are increasingly recognized as important contributors to lower functioning and poor outcomes in patients with bipolar disorder $(7,16)$, and can substantially affect survival in this group (30). Similarly, our results suggest that addressing co-occurring substance use disorders in these patients (31) and co-occurring medical conditions (32) could potentially improve overall functioning and quality of life for these patients.

In addition, there is a consistent, detrimental, and long-term impact of depression on HRQOL. Thus, treating depression in those with bipolar disorder is essential as part of the process of alleviating current symptomatology as well as improving longer-term functioning. However, the treatment of depression is difficult and recent results indicated that adjunctive antidepressant treatment may not significantly decrease depressive symptoms in those with bipolar disorder (33). It is interesting to note that several psychosocial interventions for bipolar disorder utilize strategies that directly target aspects of quality of life (e.g., family functioning, behavioral activation, improving social support) as part of a focus on management of depressive symptoms (22,31-34). The present results indicate that this more comprehensive approach may be one way to break the tie between depression and poorer functioning in patients with bipolar disorder.

This study has a number of strengths including a relatively large sample size, availability of longitudinal data on a standardized outcome measure for which population norms exist (HRQOL), and a data analytic approach that incorporates both regression and path analyses However, there are limitations to this study that warrant consideration. First, CIVIC-MD was limited to a single site; we were unable to enroll all eligible patients. Second, to reduce respondent and provider burden and reduce barriers to study entry, we relied on diagnoses based on medical record and confirmed by clinicians rather than formal structured psychiatric interview, as we did not examine other comorbidities, such as obesity. We were also unable to include assessment of the severity of anxiety symptoms. Although structured psychiatric interviews (e.g., Structured Clinical Interview for DSM-IV) can be lengthy and potentially burdensome for patients, they are considered the gold standard, and may preclude our ability to assess the effect 
of bipolar subtype (I, II) on HRQOL, and fully compare our results from CIVIC-MD with results from other previously published clinical cohorts that implemented formal diagnostic assessment. In addition, we excluded from the study individuals with acute medical conditions or acute psychiatric symptoms, and our results might have been affected by the exclusion of individuals with more severe or unstable conditions. Although we wanted to include a more generalizable patient sample, there are issues of consent for patients who are actively manic in a study or otherwise lower functioning, for which there is no potential direct benefit to the participant.

Moreover, it should be noted that the path analysis was estimated, using maximum likelihood. An ideal estimator would be weighted least squares given that the model included dichotomous variables (i.e., gender, drug use, and binge drinking) and count variables (i.e., number of co-occurring medical conditions). This type of estimation was not possible, given the requirements for very large sample sizes and less model complexity. Although the estimates in models could be slightly biased, it is important to note that the observed effects were all in the expected directions and the effect sizes were in a plausible range. The path models were included as supplemental test of the study hypotheses, complementing the strengths and weaknesses of the linear regression models. In addition, because we used the SF-12 to measure HRQOL as opposed to the full-length SF-36, we were unable to assess changes in SF-36 subscale scores (e.g., physical functioning, energy) that have been used to compare HRQOL in other bipolar disorder studies $(11,29)$. Finally, the potential generalizability of these findings may be limited to VA patients; however, these individuals often represent the largely indigent and comorbid patient populations seen in lower-income, community-based practice.

Overall, we found that, in a large sample of patients with bipolar disorder, HRQOL scores were lower than the national average. Moreover, substance use negatively affected mental HRQOL over time, and co-occurring medical conditions were associated with worse physical HRQOL over time in this group. Changes in HRQOL over time can lead to increased risk of mortality in persons with chronic conditions (13). Our findings suggest the need for multipronged treatment strategies for this group, notably interventions that address the physical as well as drug treatment needs of patients with bipolar disorder. Furthermore, applying both regression and path analysis methods can confirm the identification of potential mediators of outcome, suggesting that this approach can be used in future longitudinal studies in mental disorders.

\section{REFERENCES}

1. Lopez AD, Murray CC. The global burden of disease, 1990-2020. Nat Med 1998;4:1241-3.

2. Bauer MS, Kirk GF, Gavin C, Williford WO. Determinants of functional outcome and healthcare costs in bipolar disorder: a high-intensity follow-up study. J Affect Disord 2001;65:231-41.

3. Bryant-Comstock L, Stender M, Devercelli G. Health care utilization and costs among privately insured patients with bipolar I disorder. Bipolar Disorders 2002;4:398-405.

4. Simon GE, Unutzer J. Health care utilization and costs among patients treated for bipolar disorder in an insured population. Psychiatr Serv 1999;50:1303-8.

5. Judd LL, Akiskal HS. The prevalence and disability of bipolar spectrum disorders in the US population: re-analysis of the ECA database taking into account subthreshold cases. J Affect Disord 2003;73:123-31.

6. Kilbourne AM, Brar JS, Drayer RA, Xu X, Post EP. Cardiovascular disease and metabolic risk factors in male patients with schizophrenia, schizoaffective disorder, and bipolar disorder. Psychosomatics 2007;48:412-7.

7. Kilbourne AM, Cornelius JR, Han X, Pincus HA, Shad M, Salloum I, Conigliaro J, Haas GL. Burden of general medical conditions among individuals with bipolar disorder. Bipolar Disorders 2004;6:368-73.

8. Dean BB, Gerner D, Gerner RH. A systematic review evaluating healthrelated quality of life, work impairment, and healthcare costs and utilization in bipolar disorder. Curr Med Res Opin 2004;20:139-54.

9. Angst F, Stassen HH, Clayton PJ, Angst J. Mortality of patients with mood disorders: follow-up over 34-38 years. J Affect Disord 2002;68:167-81.

10. Michalak EE, Yatham LN, Lam RW. Quality of life in bipolar disorder: a review of the literature. Health Qual Life Outcomes 2005;3:72.

11. Maina G, Albert U, Bellodi L, Colombo C, Faravelli C, Monteleone P, Bogetto F, Cassano GB, Maj M. Health-related quality of life in euthymic bipolar disorder patients: differences between bipolar I and II subtypes. J Clin Psychiatry 2007;68:207-12.

12. Guyatt GH. A taxonomy of health status instruments. J Rheumatol 1995;22:1188-90.

13. Hays RD, Hahn H, Marshall G. Use of the SF-36 and other health-related quality of life measures to assess persons with disabilities. Arch Phys Med Rehabil 2002;83(Suppl 2):S4-9.

14. Wyrwich KW, Nienaber NA, Tierney WM, Wolinsky FD. Linking clinical relevance and statistical significance in evaluating intra-individual changes in health-related quality of life. Med Care 1999;37:469-78.

15. Hays RD, Revicki D, Coyne KS. Application of structural equation modeling to health outcomes research. Eval Health Prof 2005;28: 295-309.

16. Wells KB, Stewart A, Hays RD, Burnam MA, Rogers W, Daniels M, Berry S, Greenfield S, Ware J. The functioning and well-being of depressed patients. Results from the Medical Outcomes Study 44. JAMA 1989;262:914-9.

17. Kilbourne AM, Biswas K, Pirraglia PA, Sajatovic M, Williford WO, Bauer MS. Is the collaborative chronic care model effective for patients with bipolar disorder and co-occurring conditions? J Affect Disord 2009; 112:256-61.

18. Zhang H, Wisniewski SR, Bauer MS, Sachs GS, Thase ME. Comparisons of perceived quality of life across clinical states in bipolar disorder: data from the first 2000 Systematic Treatment Enhancement Program for Bipolar Disorder (STEP-BD) participants. Compr Psychiatry 2006;47:161-8.

19. Fenn HH, Bauer MS, Altshuler L, Evans DR, Williford WO, Kilbourne AM, Beresford TP, Kirk G, Stedman M, Fiore L. Medical comorbidity and health-related quality of life in bipolar disorder across the adult age span. J Affect Disord 2005;86:47-60.

20. Simon GE, Ludman EJ, Bauer MS, Unutzer J, Operskalski B. Long-term effectiveness and cost of a systematic care program for bipolar disorder. Arch Gen Psychiatry 2006;63:500-8.

21. Hermann BP, Vickrey B, Hays RD, Cramer J, Devinsky O, Meador K, Perrine K, Myers LW, Ellison GW. A comparison of health-related quality of life in patients with epilepsy, diabetes and multiple sclerosis. Epilepsy Res 1996;25:113-8.

22. Bauer MS, McBride L, Williford WO, Glick H, Kinosian B, Altshuler L, Beresford T, Kilbourne AM, Sajatovic M. Collaborative care for bipolar disorder: part I. Intervention and implementation in a randomized effectiveness trial. Psychiatr Serv 2006;57:927-36.

23. Kilbourne AM, Lasky E, Pincus HA, Good CB, Cooley S, Basavaraju A, Greenwald D, Fine MJ, Bauer, MS. Public-academic partnerships: the Continuous Improvement for Veterans in Care: Mood Disorders (CIVICMD) study, a VA-academic partnership. Psychiatr Serv 2008;59:483-5.

24. Ware J, Jr., Kosinski M, Keller SD. A 12-Item Short-Form Health Survey: construction of scales and preliminary tests of reliability and validity. Med Care 1996;34:220-33.

25. Bauer MS, Vojta C, Kinosian B, Altshuler L, Glick H. The Internal State Scale: replication of its discriminating abilities in a multisite, public sector sample. Bipolar Disorders 2000;2:340-6.

26. Gordon AJ, Maisto SA, McNeil M, Kraemer KL, Conigliaro RL, Kelley ME, Conigliaro J. Three questions can detect hazardous drinkers. J Fam Pract 2001;50:313-20.

27. Warner LA, Kessler RC, Hughes M, Anthony JC, Nelson CB. Prevalence 
and correlates of drug use and dependence in the United States. Arch Gen Psychiatry 1995;52:219-29.

28. Spitzer RL, Kroenke K, Williams JB. Validation and utility of a selfreport version of PRIME-MD: the PHQ primary care study. Primary care evaluation of mental disorders. Patient Health Questionnaire 2 18. JAMA 1999;282:1737-44.

29. Albert U, Rosso G, Maina G, et al. Impact of anxiety disorder comorbidity on quality of life in euthymic bipolar disorder patients: differences between bipolar I and II subtypes. J Affect Disord 2008;105:297-303.

30. Hennekens C. Increasing global burden of cardiovascular disease in general populations and patients with schizophrenia. J Clin Psychiatry 2007;68(Suppl 4):4-7.

31. Weiss RD, Griffin ML, Kolodziej ME, Greenfield SF, Najavits LM, Daley DC, Doreau HR, Hennen, JA. A randomized trial of integrated group therapy versus group drug counseling for patients with bipolar disorder and substance dependence. Am J Psychiatry 2007;164:100-7.
32. Kilbourne AM, Post EP, Nossek A, Drill L, Cooley S, Bauer MS. Improving medical and psychiatric outcomes among individuals with bipolar disorder: a randomized controlled trial. Psychiatr Serv 2008;59: $760-8$.

33. Sachs GS, Nierenberg AA, Calabrese JR, Marangell LB, Wisniewski SR, Gyulai L, Friedman ES, Bowden CL, Fossey MD, Ostacher MJ, Ketter TA, Patel J, Hauser P, Rapport D, Martinez JM, Allen MH, Miklowitz DJ, Otto MW, Dennehy EB, Thase ME. Effectiveness of adjunctive antidepressant treatment for bipolar depression. N Engl J Med 2007;356: 1711-22.

34. Miklowitz DJ, Otto MW, Frank E, Reilly-Harrington NA, Kogan JN, Sachs GS, Thase ME, Calabrese JR, Marangell LB, Ostacher MJ, Patel J, Thomas MR, Araga M, Gonzalez JM, Wisniewski SR. Intensive psychosocial intervention enhances functioning in patients with bipolar depression: results from a 9-month randomized controlled trial. Am J Psychiatry 2007;164:1340-7. 